\title{
GENERALIZED TOPOLOGICAL APPROXIMATION SPACES AND THEIR MEDICAL APPLICATIONS
}

\author{
A. S. Salama \\ Mathematics Department, Faculty of Science, Tanta University, Tanta, Egypt. \\ dr_salama75@yahoo.com
}

Received 10/2/2018

\section{Accepted 18/4/2018}

\begin{abstract}
We generalize Pawlak's approximation space to topological approximation spaces using some topological near open sets, such as regular open sets, semi-open sets, pre-open sets, $\gamma$-open sets, $\alpha$-open sets and $\beta$-open sets and others. The properties of the topological approximations space will be studied. Finally, We applied our results on medical data.
\end{abstract}

Keywords: Topological Spaces; Rough Sets; Rough Approximations; Information System; Data Reduction.

MSC: 54A05; 54B05; 54D35; $03 B 70$.

\section{Introduction}

Rough set theory, proposed by Pawlak in [1], is an extension of set theory for the study of intelligent systems characterized by inexact, uncertain or insufficient information. Many suggestions have been made for generalizing and interpreting rough sets [2-4]. Moreover, this theory may serve as a new mathematical tool to soft computing and has been successfully applied in machine learning, information sciences, expert systems, data reduction, and so on. Recently, lots of researchers are interested to generalize this theory in many fields of applications [5-9]. Several interesting and meaningful generalizations to equivalence relation have been proposed in the past, such as topological bases and subbases $[10,11,12]$. Particularly, some researchers have used coverings of the universe of discourse for establishing the generalized rough sets by coverings [13,14].

Rough set theory is a recent approach for reasoning about data. This theory depends basically on a certain topological structure and has achieved a great success in many fields of real life applications. The concept of a topological rough set given by Wiweger [15] in 1989 is one of the most important topological generalization of rough sets. In 1983 Abd El-Monsef in [16] introduced the concept of $\beta$-open sets. This paper is organized as follows:

In Section 2 we give topological basic concepts. Also, Section 3 discussed the fundamentals of rough sets and investigated the concept of topological approximation space. Section 4 is devoted to introduce medical application example. The paper's conclusion is given in Section 5 .

2. Topological Basic Concepts

More details about the following topological near open sets found in [17-20].

Definition 2. 1 [17,18] A subset $A$ of a topological space $(U, \tau)$ is called:
(1) semi-open set if $A \subseteq \operatorname{cl}(\operatorname{int}(A))$ and it is called a semiclosed set if $\operatorname{int}(\operatorname{cl}(A)) \subseteq A$

(2) pre-open set if $A \subseteq \operatorname{int}(\operatorname{cl}(A))$ and it is called a preclosed set if $c l(\operatorname{int}(A)) \subseteq A$

(3) $\alpha$-open set if $A \subseteq \operatorname{int}(\operatorname{cl}(\operatorname{int}(A)))$ and it is called a $\alpha-$ closed set if $\operatorname{cl}(\operatorname{int}(\operatorname{cl}(A))) \subseteq A$.

(4) semi-pre-open set ( $\beta$-open [1]) if $A \subseteq \operatorname{cl}(\operatorname{int}(\operatorname{cl}(A)))$ and it is called a semi-pre-closed set ( $\beta$ - closed) if $\operatorname{int}(\operatorname{cl}(\operatorname{int}(A))) \subseteq A$.

(5) regular-open set if $A=\operatorname{int}(c l(A))$ and it is called a regularclosed set if $c l(\operatorname{int}(A))=A$.

(6) semi-regular set if it both semi-open and semi-closed in $(U, \tau)$.

(7) $\delta$-closed set if $A=c l_{\delta}(A)$, where

$c_{\delta}(A)=\{x \in U: \operatorname{int}(c l(G)) \cap A \neq \phi, x \in G, G \in \tau\}$.

The semi-closure (resp. $\alpha$-closure, semi-pre-closure) of a subset $A$ of $(U, \tau)$ is the intersection of all semi-closed (resp. $\alpha$-closed, semi-pre-closed) sets that contains $A$ and is denoted by $\operatorname{scl}(A)($ resp. $\alpha-\operatorname{cl}(A), \operatorname{spcl}(A))$. The union of all semi-open subsets of $U$ is called the semi-interior of $A$ and is denoted by $\sin t(A)$.

Definition 2.2 [19,20] A subset $A$ of a topological space $(U, \tau)$ is called:

(1) generalized closed (briefly $g$ - closed) set if $\operatorname{cl}(A) \subseteq G$ whenever $A \subseteq G$ and $G \in \tau$.

(2) semi-generalized closed set (briefly $s g$ - closed) if $\operatorname{scl}(A) \subseteq G$ whenever $A \subseteq G$ and $G$ is semi-open set in $(U, \tau)$. The complement of a $s g$-closed set is called a $s g$ - open set. 
(3) generalized semi-closed set (briefly $g s-$ closed ) if $\operatorname{scl}(A) \subseteq U$ whenever $A \subseteq G$ and $G \in \tau$.

(4) $\alpha$ - generalized closed set (briefly $\alpha g$ - closed) if $\operatorname{\alpha cl}(A) \subseteq G$ whenever $A \subseteq G$ and $G \in \tau$

(5) generalized $\alpha$-closed set (briefly $g \alpha$ - closed) if $\alpha c l(A) \subseteq G$ whenever $A \subseteq G$ and $G$ is $\alpha$-open in $(U, \tau)$

(6) $g \alpha^{* *}$-closed set if $c l(A) \subseteq \operatorname{int}(c l(G)) \quad$ whenever $A \subseteq G$ and $G$ is $\alpha$-open in $(U, \tau)$.

(7) generalized semi-pre-closed (briefly $g s p$ - closed) set if $\operatorname{spcl}(A) \subseteq G$ whenever $A \subseteq G$ and $G \in \tau$.

(8) $\delta$ - generalized closed (briefly $\delta g$ - closed) set if $c l \delta(A) \subseteq U$ whenever $A \subseteq G$ and $G \in \tau$.

(9) $Q$-set if $\operatorname{int}(\operatorname{cl}(A))=c l(\operatorname{int}(A))$.

The equivalence classes of $R$ are also known as the granules, elementary sets or blocks; we will use $[x]_{R} \subseteq U$ to denote the equivalence class containing $x \in U$. In the approximation space, we consider two operators $\underline{R}(X)=\left\{x \in U:[x]_{R} \subseteq X\right\}$ and $\bar{R}(X)=\left\{x \in U:[x]_{R} \cap X \neq \phi\right\}$, called the lower approximation and upper approximation of $X \subseteq U$ respectively. Also let $\operatorname{POS}_{R}(X)=\underline{R}(X)$ denote the positive region of $X, N E G_{R}(X)=U-\bar{R}(X)$ denote the negative region of $X$ and $B N_{R}(X)=\bar{R}(X)-\underline{R}(X)$ denote the borderline region of $X$.

The degree of completeness can also be characterized by the accuracy measure, in which $|X|$ represents the cardinality of set $X$ as follows: $\alpha_{R}(X)=|\underline{R}(X)| /|\bar{R}(X)|, \bar{R}(X) \neq \phi$.

\section{Topological approximation spaces}

In this section, we introduce and investigate the concept of topological approximation space. Also, we introduce the concepts of topological lower approximation and topological upper approximation and study their properties.

Definition 3.1 Let $K=(U, R)$ be an approximation space with general relation $R$ and $\tau_{R}$ is the topology associated with $K$. Then the triple $K_{\tau}=\left(U, R, \tau_{R}\right)$ is called a topological approximation space.

Definition 3.2 Let $K_{\tau}=\left(U, R, \tau_{R}\right)$ be a topological approximation space. If $X \subseteq U$, then the topological lower approximations of $X$ are defined as follows:

(1) Semi-lower approximation of $X$, $\underline{R}_{\text {semi }}(X)=\cup\{G: G \in \operatorname{SEMI}(U), G \subseteq X\} \quad$ where $\operatorname{SEMI}(U)$ is the set of all semi-open sets in $K_{\tau}=$ $\left(U, R, \tau_{R}\right)$.

(2) Pre-lower approximation of $X$, $\underline{R}_{\text {pre }}(X)=\cup\{G: G \in \operatorname{PRE}(U), G \subseteq X\}$

where

$\operatorname{PRE}(U)$ is the set of all pre - open sets in $K_{\tau}=\left(U, R, \tau_{R}\right)$. (3) $\alpha$-lower approximation of $X$, $\underline{R}_{\alpha}(X)=\cup\{G: G \in \alpha(U), G \subseteq X\}$ where $\alpha(U)$ is the set of all $\alpha$-open sets in $K_{\tau}=\left(U, R, \tau_{R}\right)$.

(4) $\beta$-lower approximation of $X$, $\underline{R}_{\beta}(X)=\cup\{G: G \in \beta(U), G \subseteq X\}$ where $\beta(U)$ is the set of all $\beta$-open sets in $K_{\tau}=\left(U, R, \tau_{R}\right)$.
(5) Regular-lower approximation of $X$, $\underline{R}_{\text {regular }}(X)=\cup\{G: G \in R E G(U), G \subseteq X\} \quad$ where $R E G(U)$ is the set of all regular-open sets in $K_{\tau}=$ $\left(U, R, \tau_{R}\right)$.

(6) Semi-regular lower approximation of $X$,

$\underline{R}_{\text {semi-regular }}(X)=\cup\{G: G \in \operatorname{SEMI}(U)$ or $G \in C S E M I(U)$ where $\operatorname{SEMI}(U)$ and $\operatorname{CSEMI}(U)$ are the set of all semiopen sets and semi - closed sets in $K_{\tau}=\left(U, R, \tau_{R}\right)$ respectively.

(7) $\delta$-lower approximation of $X$, $\underline{R}_{\delta}(X)=\cup\{G: G \in \delta(U), G \subseteq X\}$ where $\delta(U)$ is the set of all $\delta-$ closed sets in $K_{\tau}=\left(U, R, \tau_{R}\right)$.

(8) $g$-lower approximation of $X$, $\underline{R}_{g}(X)=\cup\{G: G \in g(U), G \subseteq X\}$ where $g(U)$ is the set of all $g-$ closed sets in $K_{\tau}=\left(U, R, \tau_{R}\right)$.

(9) $s g$-lower approximation of $X$ $\underline{R}_{s g}(X)=\cup\{G: G \in \operatorname{sg}(U), G \subseteq X\}$ where $s g(U)$ is the set of all $s g$-open sets in $K_{\tau}=\left(U, R, \tau_{R}\right)$.

(10) gs-lower approximation of $X$ $\underline{R}_{g s}(X)=\cup\{G: G \in g s(U), G \subseteq X\}$ where $g s(U)$ is the set of all $g s-$ open sets in $K_{\tau}=\left(U, R, \tau_{R}\right)$.

(11) $\alpha g$-lower approximation of $X$, $\underline{R}_{\alpha g}(X)=\cup\{G: G \in \alpha g(U), G \subseteq X\}$ is the set of all $\alpha g-$ closed sets in $K_{\tau}=\left(U, R, \tau_{R}\right)$.

(12) $g \alpha$-lower approximation of $X$, $\underline{R}_{g \alpha}(X)=\cup\{G: G \in g \alpha(U), G \subseteq X\}$ where $g \alpha(U)$ is the set of all $g \alpha-$ closed sets in $K_{\tau}=\left(U, R, \tau_{R}\right)$.

(13) $g \alpha^{* *}$-lower approximation of $X$, $\underline{R}_{g \alpha * *}(X)=\cup\left\{G: G \in g \alpha^{* * *}(U), G \subseteq X\right\}$

where $g \alpha^{* *}(U)$ is the set of all $g \alpha^{* *}-$ closed sets in $K_{\tau}=$ $\left(U, R, \tau_{R}\right)$.

(14) $g s p$-lower approximation of $X$ $\underline{R}_{g s p}(X)=\cup\{G: G \in \operatorname{gsp}(U), G \subseteq X\}$

where $g s p(U)$ is the set of all $g s p-$ closed sets in $K_{\tau}=\left(U, R, \tau_{R}\right)$. (15) $\delta g$-lower approximation of $X$, $\underline{R}_{\delta g}(X)=\cup\{G: G \in \delta g(U), G \subseteq X\}$ where $\delta g(U)$ is the set of all $\delta g-$ closed sets in $K_{\tau}=\left(U, R, \tau_{R}\right)$. (16) Q-lower approximation of $X$ $\underline{R}_{Q}(X)=\cup\{G: G \in Q(U), G \subseteq X\}$ where $Q(U)$ is the set of all $Q-$ sets in $K_{\tau}=\left(U, R, \tau_{R}\right)$.

Definition 3.3 Let $K_{\tau}=\left(U, R, \tau_{R}\right)$ be a topological approximation space. If $X \subseteq U$, then the topological upper approximations of $X$ are defined as follows:
(1) Semi-upper approximation of $X$,
$\bar{R}_{\text {semi }}(X)=\cup\{F: F \in \operatorname{CSEMI}(U), F \cap X \neq \varphi\}$

where $\operatorname{CSEMI}(U)$ is the set of all semi-closed sets in $K_{\tau}=$ $\left(U, R, \tau_{R}\right)$.

(2) Pre-upper approximation of $X$, $\bar{R}_{\text {pre }}(X)=\cup\{F: F \in C P R E(U), F \cap X \neq \varphi\}$ 
where $C P R E(U)$ is the set of all pre - closed sets in $K_{\tau}=$ $\left(U, R, \tau_{R}\right)$.

(3) $\quad \alpha$-upper approximation of $X$, $\bar{R}_{\alpha}(X)=\cup\{F: F \in C \alpha(U), F \cap X \neq \varphi\} \quad$ where $C \alpha(U)$ is the set of all $\alpha-$ closed sets in $K_{\tau}=\left(U, R, \tau_{R}\right)$. (4) $\quad \beta$-upper approximation of $X$ $\bar{R}_{\beta}(X)=\cup\{F: F \in C \beta(U), F \cap X \neq \varphi\}$ $C \beta(U)$ is the set of all $\beta-$ closed sets in $K_{\tau}=\left(U, R, \tau_{R}\right)$.

(5) Regular-upper approximation of $X$,

$\bar{R}_{\text {regular }}(X)=\cup\{F: F \in C R E G(U), F \cap X \neq \varphi\}$ where $C R E G(U)$ is the set of all regular - closed sets in $K_{\tau}=\left(U, R, \tau_{R}\right)$.

(6) Semi-regular upper approximation of $X$,

$\bar{R}_{\text {semi-regular }}(X)=\cup\{F: F \in C S E M I(U), F \cap X \neq c$ where $\operatorname{CSEMI}(U)$ and $\operatorname{CSEMI}(U)$ is the set of all semi closed sets in $K_{\tau}=\left(U, R, \tau_{R}\right)$.

(7) $\delta$-upper approximation of $X$, $\bar{R}_{\delta}(X)=\cup\{F: F \in \delta(U), F \cap X \neq \varphi\}$ where $\delta(U)$ is the set of all $\delta$-closed sets in $K_{\tau}=\left(U, R, \tau_{R}\right)$.

(8) $g$-upper approximation of $X$, $\bar{R}_{g}(X)=\cup\{F: F \in g(U), F \cap X \neq \varphi\}$ where $g(U)$ is the set of all $g-$ closed sets in $K_{\tau}=\left(U, R, \tau_{R}\right)$.

(9) $s g$-upper approximation of $X$ $\bar{R}_{s g}(X)=\cup\{F: F \in C s g(U), F \cap X \neq \varphi\} \quad$ where $\operatorname{Csg}(U)$ is the set of all $s g$ - closed sets in $K_{\tau}=\left(U, R, \tau_{R}\right)$. (10) gs-upper approximation of $X$ $\bar{R}_{g s}(X)=\cup\{F: F \in C g s(U), F \cap X \neq \varphi\} \quad$ where $\operatorname{Cgs}(U)$ is the set of all $g s-$ closed sets in $K_{\tau}=\left(U, R, \tau_{R}\right)$. (11) $\alpha g$-upper approximation of $X$, $\bar{R}_{\alpha g}(X)=\cup\{F: F \in \alpha g(U), F \cap X \neq \varphi\}$ where $\alpha g(U)$ is the set of all $\alpha g-$ closed sets in $K_{\tau}=\left(U, R, \tau_{R}\right)$. (12) $g \alpha$-upper approximation of $X$, $\bar{R}_{g \alpha}(X)=\cup\{F: F \in g \alpha(U), F \cap X \neq \varphi\}$ where $g \alpha(U)$ is the set of all $g \alpha-$ closed sets in $K_{\tau}=\left(U, R, \tau_{R}\right)$. (13) $g \alpha^{* *}$-upper approximation of $X$, $\bar{R}_{g \alpha * *}(X)=\cup\left\{F: F \in g \alpha^{* * *}(U), F \cap X \neq \varphi\right\}_{\text {where }}$ $g \alpha^{* *}(U)$ is the set of all $g \alpha^{* *}-$ closed sets in $K_{\tau}=$ $\left(U, R, \tau_{R}\right)$.

(14) gsp-upper approximation of $X$ $\bar{R}_{g s p}(X)=\cup\{F: F \in g s p(U), F \cap X \neq \varphi\}$ where $g s p(U)$ is the set of all $g s p-$ closed sets in $K_{\tau}=\left(U, R, \tau_{R}\right)$. (15) $\delta g$-upper approximation of $X$, $\bar{R}_{\delta g}(X)=\cup\{F: F \in \delta g(U), F \cap X \neq \varphi\} \quad$ where $\delta g(U)$ is the set of all $\delta g-$ closed sets in $K_{\tau}=\left(U, R, \tau_{R}\right)$. (16) Q-upper approximation of $X$ $\bar{R}_{Q}(X)=\cup\{F: F \in Q(U), F \cap X \neq \varphi\}$

$Q(U)$ is the set of all $Q-$ sets in $K_{\tau}=\left(U, R, \tau_{R}\right)$.

Motivation for topological rough set theory has come from the need to represent subsets of a universe in terms of topological classes of the topological base generated by the general binary relation defined on the universe. That base characterizes a topological space, called topological approximation space $K_{\tau}=\left(U, R, \tau_{R}\right)$. The topological classes of $R$ are also known as the topological granules, topological elementary sets or topological blocks; we will use $G_{x m} \in \tau$, $m \in\{$ semi, pre, $\alpha, \beta$, regular, semi -

regular $\left.\delta, g, s g, g s, \alpha g, g \alpha, g \alpha^{* *}, g s p, \delta g, Q\right\}$ to denote the topological class containing $x \in U$. In the topological approximation space, we consider two operators $\underline{R}_{m}(X)=\left\{x \in U: G_{x m} \subseteq X\right\}$

and

$\bar{R}_{m}(X)=\left\{x \in U: G_{x m} \cap X \neq \phi\right\}$

called the topological lower approximation and topological upper approximation of $X \subseteq U$ respectively. Also let $\operatorname{POS}_{m}(X)=\underline{R}_{m}(X)$

denote the topological positive region of $X,{ }_{m}^{N E G}(X)=U-\bar{R}_{m}(X)$ topological negative region of $X$ and $\operatorname{BND}_{m}(X)=\bar{R}_{m}(X)-\underline{R}_{m}(X)$ borderline region of $X$.

denote the topological

The degree of topological completeness can also be characterized by the topological accuracy measure, in which $|X|$ represents the cardinality of set $X$ as follows:

$$
\alpha_{m}(X)=\frac{\left|\underline{R}_{m}(X)\right|}{\left|\bar{R}_{m}(X)\right|} \text {, where } X \neq \phi
$$

Example 3. 1 Let $U=\{a, b, c, d\}$ be a universe and a relation $R$ defined by $R=$

$\{(a, a),(a, c),(a, d),(b, b),(b, d),(c, a),(c, b),(c, d),(d, a)\}$ , thus $a R=\{a, c, d\}, b R=\{b, d\}, c R=\{a, b, d\}$ and $d R=\{a\}$. Then the topology associated with this relation $R$ is $\tau_{R}=\{U, \varphi,\{a\},\{d\},\{a, d\},\{b, d\},\{a, b, d\},\{a, c, d\}\}$ and $\beta(U)=$

$\{U, \varphi,\{a\},\{d\},\{a, c\},\{a, d\},\{b, d\},\{c, d\},\{a, b, d\},\{a, c, d\},\{b, c, d\}\}$

. So $\left(U, R, \tau_{R}\right)$ is a topological approximation space using

$\beta$-open sets.

Let $\left(U, R, \tau_{R}\right)$ be a topological approximation space. The Universe $U$ can be divided into many regions with respect to any $X \subseteq U$ and with respect to any

$m \in\{$ semi, pre, $\alpha, \beta$, regular, semi-regular, $\delta, \mathrm{g}, \mathrm{sg}, \mathrm{gs}, \alpha \mathrm{g}, \mathrm{g} \alpha$, $\left.\mathrm{g} \alpha^{\wedge}(* *), \mathrm{gsp}, \delta \mathrm{g}, \mathrm{Q}\right\}$ as follows:

Let $\left(U, R, \tau_{R}\right)$ be a topological approximation space. For any $m \in\{$ semi, pre, $\alpha, \beta$, regular, semi-regular, $\delta$, g, sg, gs, $\left.\alpha \mathrm{g}, \mathrm{g} \alpha, \mathrm{g} \alpha^{\wedge}(* *), \mathrm{gsp}, \delta \mathrm{g}, \mathrm{Q}\right\}$ and for any subset $X \subseteq U$ we define the following memberships:

$\mathrm{x}$ belongs strong to $\mathrm{X}$ if $x \in \underline{R}(X)$,x belongs weak to $\mathrm{X}$ if $x \in \bar{R}(X)$,

x belongs m-strong to $X$ if $x \in \underline{R}_{m}(X)$, x belongs mweak to $X$ if $x \in \bar{R}_{m}(X)$,

Example 3.2 Let $U=\{a, b, c, d\}$ be a universe and a relation $\mathrm{R}$ defined by $\mathrm{R}=\{(\mathrm{a}, \mathrm{a}),(\mathrm{d}, \mathrm{c}),(\mathrm{d}, \mathrm{d}),(\mathrm{c}, \mathrm{a}),(\mathrm{c}, \mathrm{d}),(\mathrm{c}, \mathrm{c})\}$, thus $\mathrm{aR}=\{\mathrm{a}\}, \mathrm{bR}=\varphi, \mathrm{cR}=\{\mathrm{a}, \mathrm{c}, \mathrm{d}\}$ and $d \mathrm{R}=\{\mathrm{c}, \mathrm{d}\}$. Then the 
topology associated with this relation is $\tau \mathrm{R}=\{\mathrm{U}, \varphi,\{\mathrm{a}\},\{\mathrm{c}$, $\mathrm{d}\},\{\mathrm{a}, \mathrm{c}, \mathrm{d}\}\}$. So $\left(U, R, \tau_{R}\right)$ is a topological approximation space. Let $X=\{b, c, d\}$, we have $b$ is $m$-strong belongs to $X$ but $\mathrm{b}$ is not strong belongs to $\mathrm{X}$. Also, let $\mathrm{Y}=\{\mathrm{c}\}$ be another subset of $\mathrm{U}$. Then we have $\mathrm{d}$ is weak belongs to $Y$ but $\mathrm{d}$ is not m-weak belongs to $\mathrm{Y}$.

The degree of topological completeness can also be characterized by the topological accuracy measure (maccuracy), in which $|X|$ represents the cardinality of set $X$ as follows:

$$
\alpha_{m}(X)=\frac{\left|\underline{R}_{m}(X)\right|}{\left|\bar{R}_{m}(X)\right|}, \text { where } X \neq \phi
$$

According to Example 3.1, Table 1 showing the differences among the degree of Pawlak's accuracy measure $\alpha(X)$ and $\beta$ accuracy measure $\alpha \beta(\mathrm{X})$ for some subsets of $\mathrm{U}$ if we take $\mathrm{m}=\beta$.

\begin{tabular}{|l|l|l|}
\hline \multicolumn{1}{|c|}{$X$} & \multicolumn{1}{c|}{$\alpha(X)$ (Pawlak) } & \multicolumn{1}{c|}{$\alpha_{\beta}(X)$} \\
\hline$\{\mathrm{a}\}$ & $50 \%$ & $100 \%$ \\
\hline$\{\mathrm{a}, \mathrm{c}\}$ & $50 \%$ & $100 \%$ \\
\hline$\{\mathrm{b}, \mathrm{d}\}$ & $33.3 \%$ & $100 \%$ \\
\hline$\{\mathrm{b}, \mathrm{c}, \mathrm{d}\}$ & $66.6 \%$ & $100 \%$ \\
\hline
\end{tabular}

\section{Table 1:Comparision between accuracy measures}

The $\beta$-accuracy measure of the class of all $\beta$-open sets is accurate than the others measures and the following example and its followed diagram illustrate this fact.

Example 3.3 Let $U=\{a, b, c, d\}$ be a universe and for some relations we have the topology $\tau_{\mathrm{R}}=\{\mathrm{U}, \phi,\{\mathrm{d}\},\{\mathrm{a}, \mathrm{b}\},\{\mathrm{a}, \mathrm{b}, \mathrm{d}\}\}$

So $(\mathrm{U}, \mathrm{R}, \tau \mathrm{R})$ is a topological approximation space. The we have the following knowledge bases:

$\operatorname{REG}(\mathrm{U})=\{\mathrm{U}, \varphi,\{\mathrm{d}\},\{\mathrm{a}, \mathrm{b}\}\}$,

$\operatorname{SEMI}(\mathrm{U})=\{\mathrm{U}, \varphi,\{\mathrm{d}\},\{\mathrm{a}, \mathrm{b}\},\{\mathrm{c}, \mathrm{d}\},\{\mathrm{a}, \mathrm{b}, \mathrm{c}\},\{\mathrm{a}, \mathrm{b}, \mathrm{d}\}\}$,

$\operatorname{PRE}(\mathrm{U})=\{\mathrm{U}, \varphi,\{\mathrm{a}\},\{\mathrm{b}\},\{\mathrm{d}\},\{\mathrm{a}, \mathrm{d}\},\{\mathrm{a}, \mathrm{b}\},\{\mathrm{b}, \mathrm{d}\},\{\mathrm{a}, \mathrm{c}, \mathrm{d}\},\{\mathrm{a}, \mathrm{b}$, d $\},\{b, c, d\}\}$,

$\alpha(\mathrm{U})=\{\mathrm{U}, \varphi,\{\mathrm{d}\},\{\mathrm{a}, \mathrm{b}\},\{\mathrm{a}, \mathrm{b}, \mathrm{d}\}\}$,

$\beta(\mathrm{U})=\{\mathrm{U}, \varphi,\{\mathrm{a}\},\{\mathrm{b}\},\{\mathrm{d}\},\{\mathrm{a}, \mathrm{d}\},\{\mathrm{a}, \mathrm{b}\},\{\mathrm{a}, \mathrm{c}\},\{\mathrm{b}, \mathrm{d}\},\{\mathrm{b}, \mathrm{c}\}$,

$\{c, d\},\{a, b, c\},\{a, c, d\},\{a, b, d\},\{b, c, d\}\}$,

Proposition 3.2 For any topological approximation space $K_{\tau}=\left(U, R, \tau_{R}\right)$,the following are hold for any $X, Y \subseteq U$

$\underline{R}_{m}(X \cup Y) \supseteq \underline{R}_{m}(X) \cup \underline{R}_{m}(Y)$

$\bar{R}_{m}(X \cup Y) \supseteq \bar{R}_{m}(X) \cup \underline{R}_{m}(Y)$

$\underline{R}_{m}(X \cap Y) \subseteq \underline{R}_{m}(X) \cap \underline{R}_{m}(Y)$

$\bar{R}_{m}(X \cap Y) \subseteq \bar{R}_{m}(X) \cap \underline{R}_{m}(Y)$

Proof: We prove Part 1 and other parts are similar to it.

Since we have $X \subseteq X \cup Y$ and $Y \subseteq X \cup Y$. Then $\underline{R}_{m}(X) \subseteq \underline{R}_{m}(X \cup Y)$ and $\underline{R}_{m}(Y) \subseteq \underline{R}_{m}(X \cup Y)$

then $\underline{R}_{m}(X \cup Y) \supseteq \underline{R}_{m}(X) \cup \underline{R}_{m}(Y)$

\section{Medecal Applications Example}

The basic concepts about information systems can be found in $[1,2,3,15,20]$.

Table 2 is shown the patients information system and respective symptoms, and the data are of the discrete type.

\begin{tabular}{|l|c|c|c|c|}
\hline \multirow{2}{*}{$\begin{array}{l}\text { Patients } \\
\text { (U) }\end{array}$} & \multicolumn{2}{|c|}{ Conditional Attributes ( C) } & $\begin{array}{c}\text { Decision } \\
\text { (D) }\end{array}$ \\
\cline { 2 - 5 } & Temperature & Flu & Headache & Dengue \\
\hline u1 & Normal & No & No & No \\
\hline u2 & High & No & No & No \\
\hline u3 & Very High & No & No & Yes \\
\hline u4 & High & No & Yes & Yes \\
\hline u5 & Very High & No & Yes & Yes \\
\hline u6 & High & Yes & Yes & Yes \\
\hline u7 & Very High & Yes & Yes & Yes \\
\hline u8 & High & No & No & No \\
\hline u9 & Very High & Yes & No & Yes \\
\hline u10 & High & Yes & No & No \\
\hline u11 & Very High & Yes & No & No \\
\hline u12 & Normal & No & Yes & No \\
\hline u13 & High & No & Yes & Yes \\
\hline u14 & Normal & No & Yes & No \\
\hline u15 & Normal & Yes & Yes & No \\
\hline u16 & Normal & Yes & No & No \\
\hline u17 & High & Yes & No & No \\
\hline u18 & Very High & Yes & Yes & Yes \\
\hline u19 & Normal & Yes & No & No \\
\hline u20 & Normal & No & Yes & No \\
\hline
\end{tabular}

Table 2: Patients Information System

Where, $U$ is the set of patients (objects) or registrations of the system, such that $U=$ $\{u 1, u 2, u 3, u 4, u 5, u 6, u 7, u 8, u 9, u 10, u 11, u 12, u 13, u 14, u 15, u 16, u$ . The set of conditional attributes is represented by $\mathrm{C}=\{$ Temperature, Flu, Headache $\}$ and the set $\mathrm{D}$ represented the decision attribute, where $\mathrm{D}=\{$ Dengue $\}$.

According to Pawlak's rough set approach Table 2 is reduced to Table 3 below.

\begin{tabular}{|l|c|c|c|l|}
\hline \multirow{2}{*}{$\begin{array}{l}\text { Patients } \\
\text { (U) }\end{array}$} & \multicolumn{3}{|c|}{ Conditional Attributes ( C) } & $\begin{array}{l}\text { Decision } \\
\text { (D) }\end{array}$ \\
\cline { 2 - 5 } & Temperature & Flu & Headache & Dengue \\
\hline u1 & Normal & No & No & No \\
\hline u3 & Very High & No & No & Yes \\
\hline u4 & High & No & Yes & Yes \\
\hline
\end{tabular}

Table 3: Reduced of Table 1 using Pawlak's rough approach According to Table 2 above we have the following Decision rules:

r1: If patient Temperature $=$ Normal and Flu $=N o$ and Headache $=$ No Then Dengue $=N o$,

r2: If patient Temperature = Very High and $\mathrm{Flu}=\mathrm{No}$ and Headache $=$ No Then Dengue = Yes,

r3: If patient Temperature $=H i g h$ and Flu $=N o$ and Headache $=$ Yes Then Dengue = Yes.

According to $m$-rough set approach Table 2 is reduced to Table 4 below. 


\begin{tabular}{|l|c|c|l|}
\hline \multirow{2}{*}{$\begin{array}{l}\text { Patients } \\
\text { (U) }\end{array}$} & \multicolumn{2}{|c|}{ Conditional Attributes ( C) } & $\begin{array}{l}\text { Decision } \\
\text { (D) }\end{array}$ \\
\cline { 2 - 4 } & Temperature & Headache & Dengue \\
\hline u1 & Normal & No & No \\
\hline u2 & High & No & No \\
\hline u3 & Very High & No & Yes \\
\hline u4 & High & Yes & Yes \\
\hline u5 & Very High & Yes & Yes \\
\hline u6 & High & Yes & Yes \\
\hline u7 & Very High & Yes & Yes \\
\hline u8 & High & No & No \\
\hline u10 & High & No & No \\
\hline u12 & Normal & Yes & No \\
\hline u15 & Normal & Yes & No \\
\hline u16 & Normal & No & No \\
\hline u19 & Normal & No & No \\
\hline
\end{tabular}

Table 4: Reduced of Table 2 using $m$-rough set approach According to Table 4 above we have the following elimination decision rules:

r1: If patient Temperature $=$ Normal and Headache $=$ No Then Dengue $=$ No,

r2: If patient Temperature $=$ High and Headache $=$ No Then Dengue $=$ No,

r3: If patient Temperature = VeryHigh and

Headache $=N o$ Then Dengue $=$ Yes,

r4: If patient Temperature $=$ High and Headache $=$ Yes Then Dengue = Yes,

r5: If patient Temperature = VeryHigh and

Headache $=$ Yes Then Dengue = Yes,

$r 10$ :If patient Temperature $=$ Normal and Headache $=$ Yes Then Dengue $=N o$,

Using our approach we obtained rich decision rules that support many fields of applications in computer science such as artificial intelligence.

\section{Conclusion}

The objectives of this work are to study a new alternative methods of data mining. It is about the rough sets theory and its generalizations to topological notions used for the mining of decision rules. The advantage of these generalizations are a mathematic base of rough sets and the possibility of mathematic description of this problem.

\section{References}

[1] Z. Pawlak, Rough Sets, Theoretical Aspects of Reasoning about Data, Kluwer Academic, Boston, 1991.

[2] A. S. Salama, O. G. El-Barbary, El Sayed Atlam; Granular information retrieval using neighbourhood systems, Mathematical Methods\& Applied Sciences, 2017;1-17.

[3] A. S. Salama and O. G. El-Barbary; Topological Approach to Retrieve Missing Values in Incomplete Information systems, Journal of The Egyptian Mathematical Society. Volume 25, Issue 4, October 2017, Pages 419-423.

[4] A. S. Salama and O. G. El-Barbary; Applications of Some Topological Near Open Sets to Knowledge Discovery, International Journal of Advanced Computer Science and Applications, Vol. 7, No. 1, 2016.

[5] A. S. Salama; Generalizations of Rough Sets Using two Topological Spaces with Medical Applications,
INFORMATION Journal - Japan 2016. Vol. 19 No. 7(A) pp. 2425-2440.

[6] A. S. Salama and O. G. El-Barbary; Future Applications of Topology by Computer Programming, Life Science Journal 2014; 11(4).

[7] A. S. Salama and O. G. El-Barbary; A New Technique for Building Automatic Arabic Field Dictionary, Pensee Journal, 2014.

[8] A. S. Salama and O. G. El-Barbary; New Topological Approaches for Data Granulation, Journal of Software Engineering and Applications, 2013, 6, 1-6.

[9] A. S. Salama and O. G. El-Barbary; Multi topological approximations of rough set theory, Int. J. Granular Computing, Rough Sets and Intelligent Systems, Vol. 3, No. 1, (2013) 1.

[10] A. S. Salama and O. G. El-Barbary; New Topological Approach to Vocabulary Mining and Document Classification, Life Science Journal 2014; 11(5).

[11] A. S. Salama; Topological Solution for Missing Attribute Values in Incomplete Information Tables, Information Sciences 180,(2010) 631-639.

[12] W. Zhu, Topological approaches to covering rough sets, Information Sciences 177 (2007) 1499-1508.

[13] W. Zhu, Relationship between generalized rough sets based on binary relation and covering, Information Sciences 179 (2009) 210-225.

[14] G. Liu and Y. Sai, A comparison of two types of rough sets induced by coverings, International Journal of Approximate Reasoning 50 (2009) 521-528.

[15] A. Wiweger, On topological rough sets, Bull. Pol. Acad. Math., 37 (1989) 89-93.

[16] M. E. Abd El-Monsef, S. N. El-Deeb and R. A. Mahmoud, _-open sets and _-continuous mappings, Bull. Fac. Assiut Uni. 12 (1) (1983) 77-90.

[17] E. Hatir and T. Noiri, Decompositions of continuity and complete continuity, Acta Math. Hungary 11 (3) 4 (2006) 281-287.

[18] Bin Chen; Topological Structures on Covering-based Rough Sets, INFORMATION, Vol.16, No.6 (A), June, 2013, pp.3335-3342.

[19] E. Hatir and T. Noiri, On $\beta$-continuous functions, Chaos, Solitons and Fractals 42 (2009) 205-211.

[20] J. Kelley, General Topology, Van Nostrand Company, (1955). 